\title{
Structural Evolution from Noninterpenetrated to Interpenetrated Thorium-Organic Frameworks Exhibiting High Propyne Storage
}

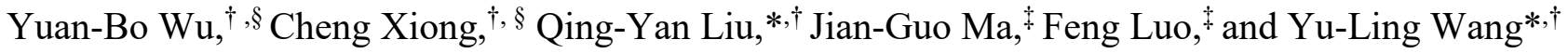

${ }^{\dagger}$ College of Chemistry and Chemical Engineering, Key Laboratory of Functional Small Molecules for Ministry of Education, Jiangxi Normal University, Nanchang 330022, China.

* State Key Laboratory of Nuclear Resources and Environment, East China University of Technology, Nanchang 330013, Jiangxi, P. R. China. ${ }^{\S}$ These authors contributed equally.

\section{Supporting Information}

\section{Experimental Section}

Physical Measurements. FT-IR spectrum was recorded from KBr disc on a Perkin-Elmer Spectrum One FT-IR spectrometer ranging from 400 to $4000 \mathrm{~cm}^{-1}$. Thermogravimetric analyses were performed under a nitrogen atmosphere with a heating rate of $10{ }^{\circ} \mathrm{C} / \mathrm{min}$ using a PE Diamond thermogravimetric analyser. Powder X-ray diffraction analyses were performed on a Rigaku Miniflex 600 diffractometer with $\mathrm{Cu}-\mathrm{K} \alpha$ radiation $(\lambda=1.5418 \AA)$.

Isosteric heat of adsorption. The isosteric heat of adsorption, $Q_{\mathrm{st}}$, is defined as $Q_{s t}=R T^{2}\left(\frac{\partial \ln p}{\partial T}\right)_{q}$ equation (1)

The $\mathrm{C}_{3} \mathrm{H}_{4}$ adsorption data were fitted using a Virial-type expression (Figure S7). ${ }^{1}$ Then the $Q_{\text {st }}$ values for $\mathrm{C}_{3} \mathrm{H}_{4}$ were calculated based on the fitting parameters with the above expression (Figure S7).

Grand canonical Monte Carlo (GCMC) simulation and Density Functional Theory (DFT) Calculation. All the GCMC simulation was performed using the Materials Studio 5.5 package. The adsorption site of $\mathrm{C}_{3} \mathrm{H}_{4}$ at $298 \mathrm{~K}$ was obtained from GCMC simulations through the fixed loading 
task in the Sorption module. The host framework and the guest molecules were both regarded as rigid. The simulation box consisted of eight unit cell and the Metropolis method based on the universal forcefield (UFF) was used. The $Q_{\mathrm{Eq}}$ derived charges and the ESP charges derived by DFT were employed to the host framework and guest atoms, respectively. The cutoff radius was chosen as $15.5 \AA$ for the Lennard-Jones (LJ) potential, and the equilibration steps and production steps were both set as $5 \times 10^{6}$. We first optimized the Th-TFBPDC structure using the DFT method with periodic boundary. The widely used generalized gradient approximation (GGA) with the Perdew-Burke-Ernzerhof (PBE) functional and the double numerical plus d-functions (DND) basis set were used. An accurate DFT Semi-core Pseudopots (DSPP) was employed for the metal atoms. For all the DFT calculations, the energy, gradient and displacement convergence criterions were set as $1 \times 10^{-5} \mathrm{Ha}, 2 \times 10^{-3} \AA$ and $5 \times 10^{-3} \AA$, respectively.

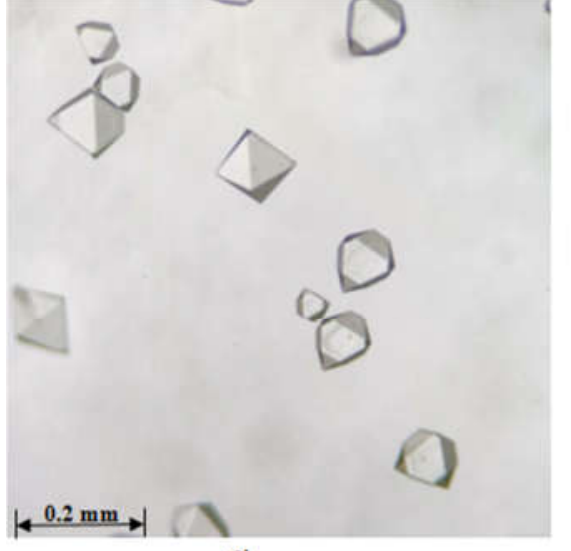

$6 \mathrm{~h}$ Th-TFBPDC

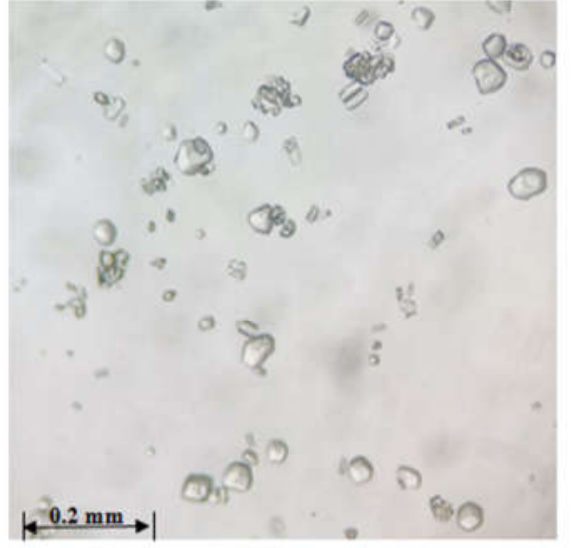

$18 \mathrm{~h}$

The co-existed of Th-TFBPDC and Th-TFBPDC-i

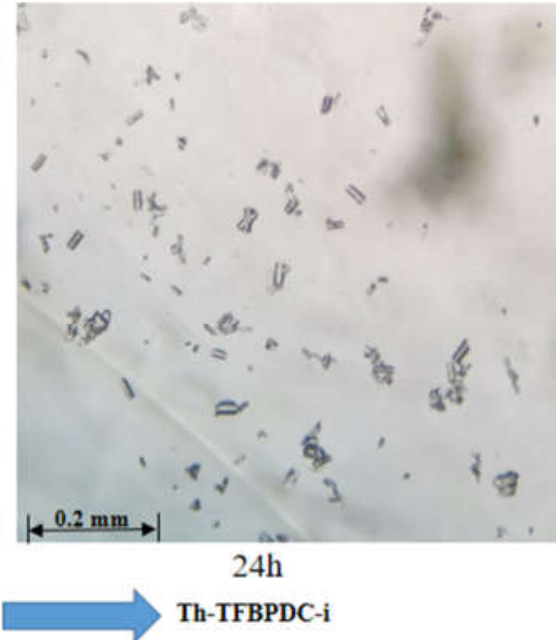

Figure S1. The optical photographs of the crystals at different reaction times.

(a)

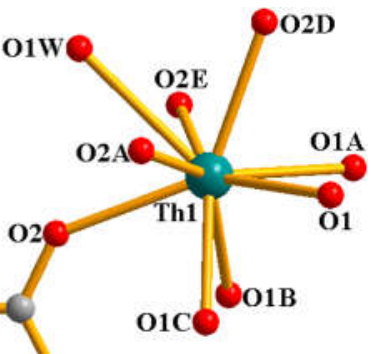




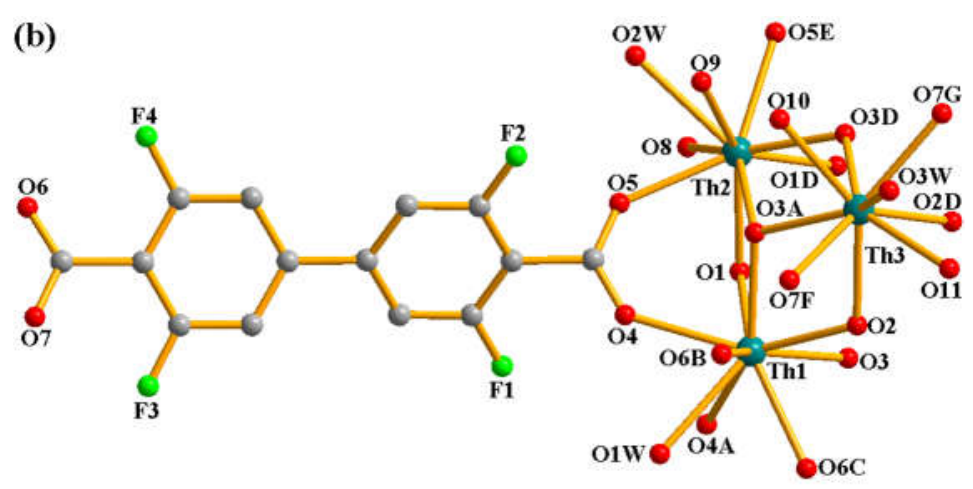

Figure S2. Coordination environments of Th(IV) atoms in Th-TFBPDC (a) to Th-TFBPDC-i (b).
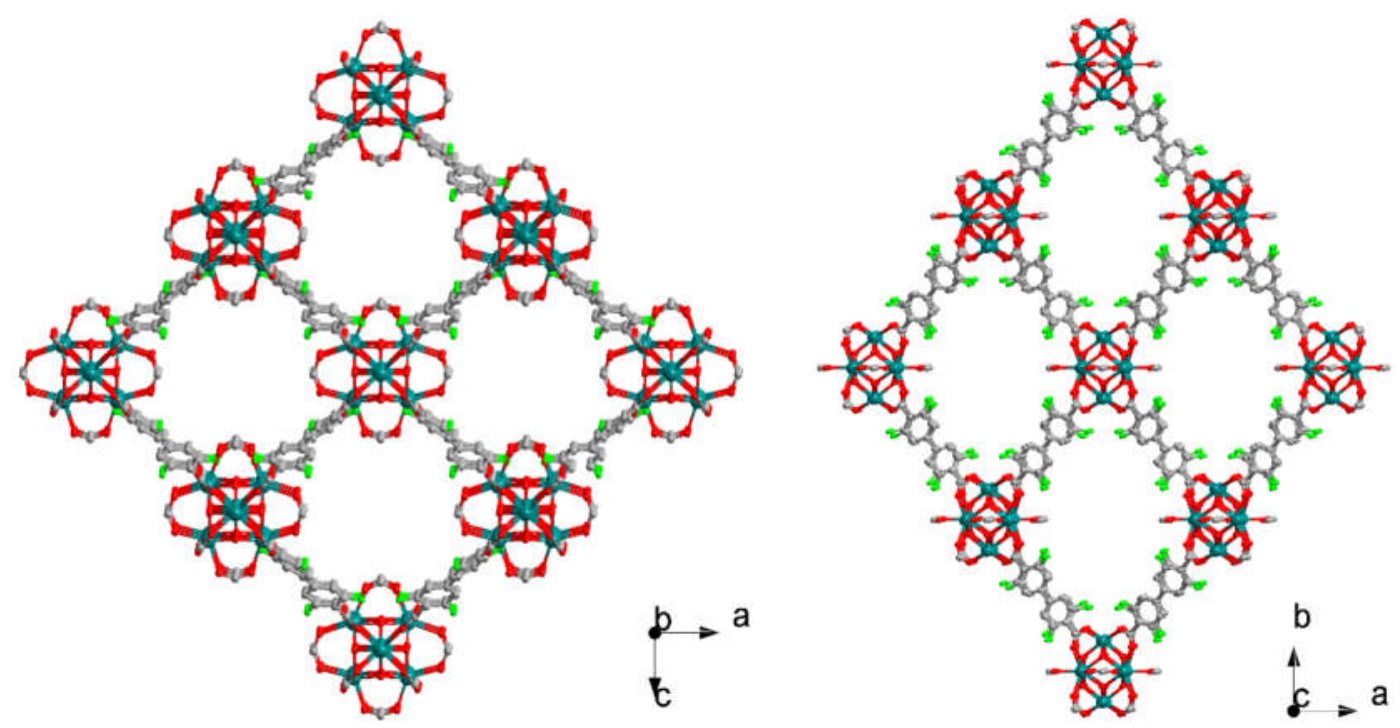

Figure S3. The single frameworks for Th-TFBPDC-i (along $b$ and $c$ axis).
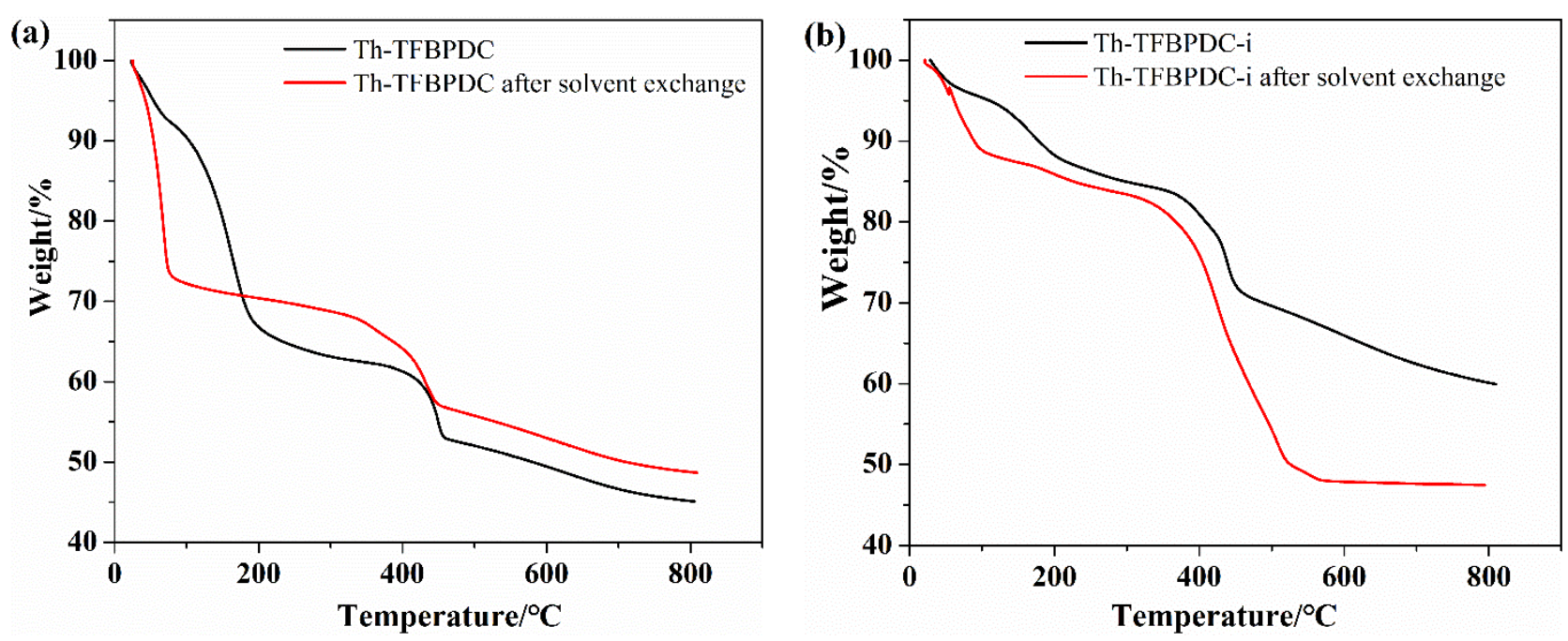

Figure S4. TGA curves. 

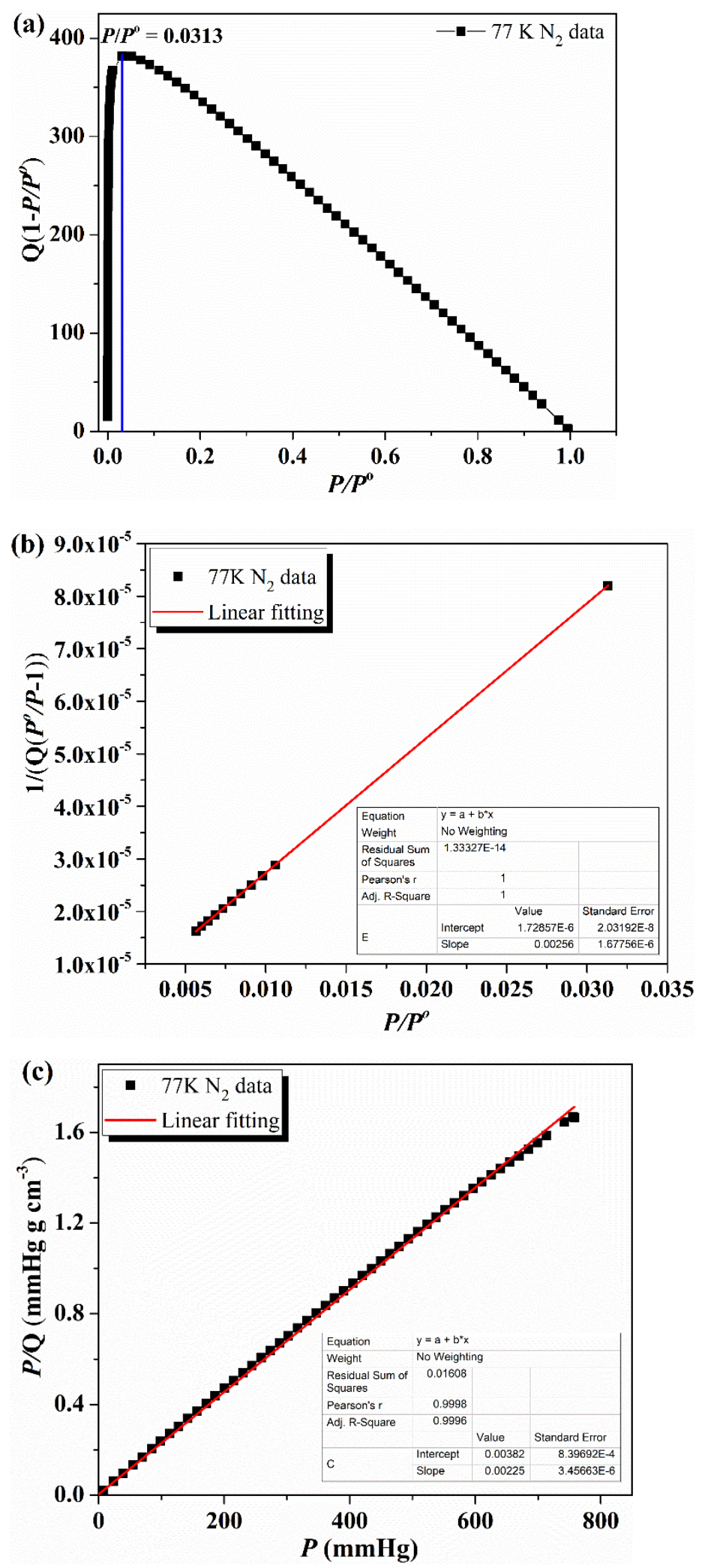

$S_{\mathrm{BET}}=\left(6.023 \times 10^{23} \times 0.162 \times 10^{-18}\right) /\left(\left(1.72857 \times 10^{-6}+0.00256\right) \times 22414\right)=1699.3 \mathrm{~m}^{2} \mathrm{~g}^{-1} \quad($ equation 2$)$ $S_{\text {Langmuir }}=\left(6.023 \times 10^{23} \times 0.162 \times 10^{-18}\right) /(22414 \times 0.00225)=1934.76 \mathrm{~m}^{2} \mathrm{~g}^{-1} \quad$ (equation 3)

Figure S5. The consistency plot (a), BET surface area plot (b), and Langmuir surface area plot (c) for Th-TFBPDC. 

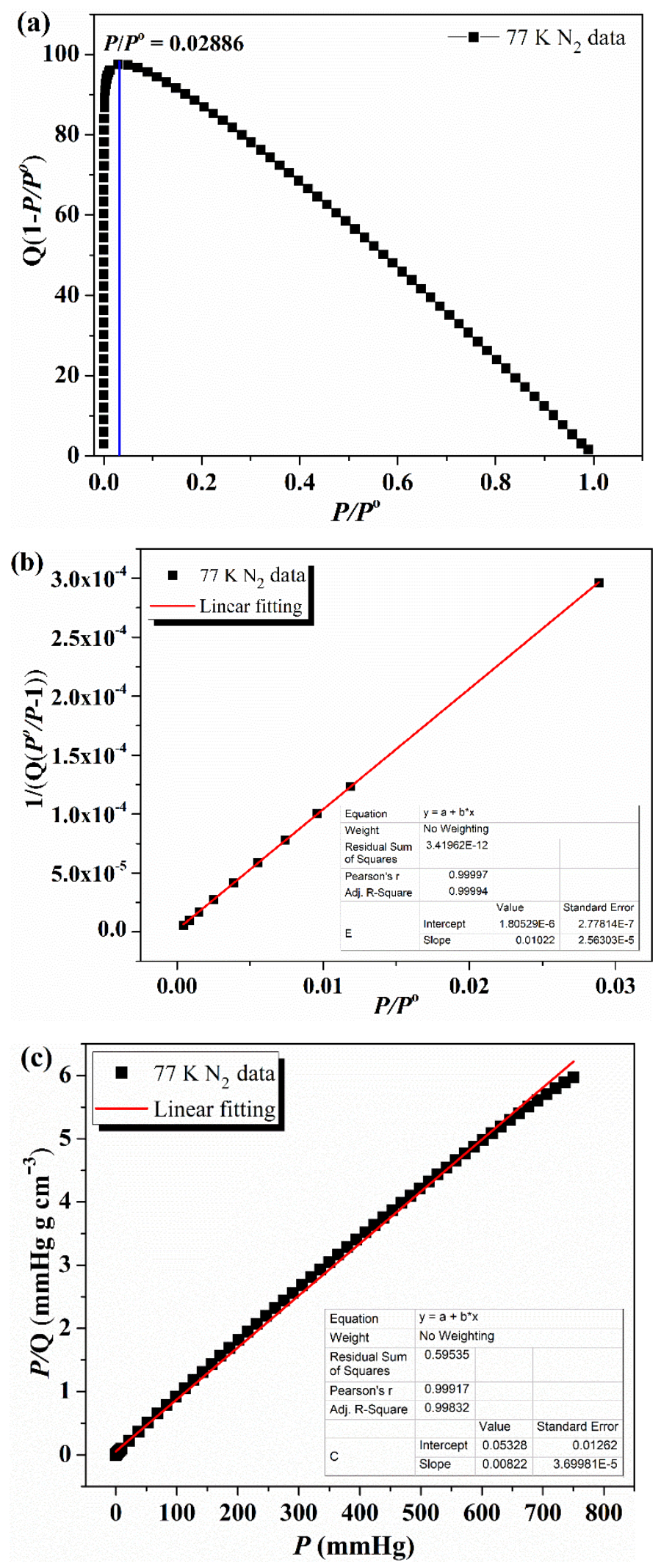

$S_{\mathrm{BET}}=\left(6.023 \times 10^{23} \times 0.162 \times 10^{-18}\right) /\left(\left(1.80529 \times 10^{-6}+0.01022\right) \times 22414\right)=425.8 \mathrm{~m}^{2} \mathrm{~g}^{-1} \quad($ equation 4$)$ $S_{\text {Langmuir }}=\left(6.023 \times 10^{23} \times 0.162 \times 10^{-18}\right) /(22414 \times 0.00225)=529.5 \mathrm{~m}^{2} \mathrm{~g}^{-1} \quad$ (equation 5)

Figure S6. The consistency plot (a), BET surface area plot (b), and Langmuir surface area plot (c) for Th-TFBPDC-i. 

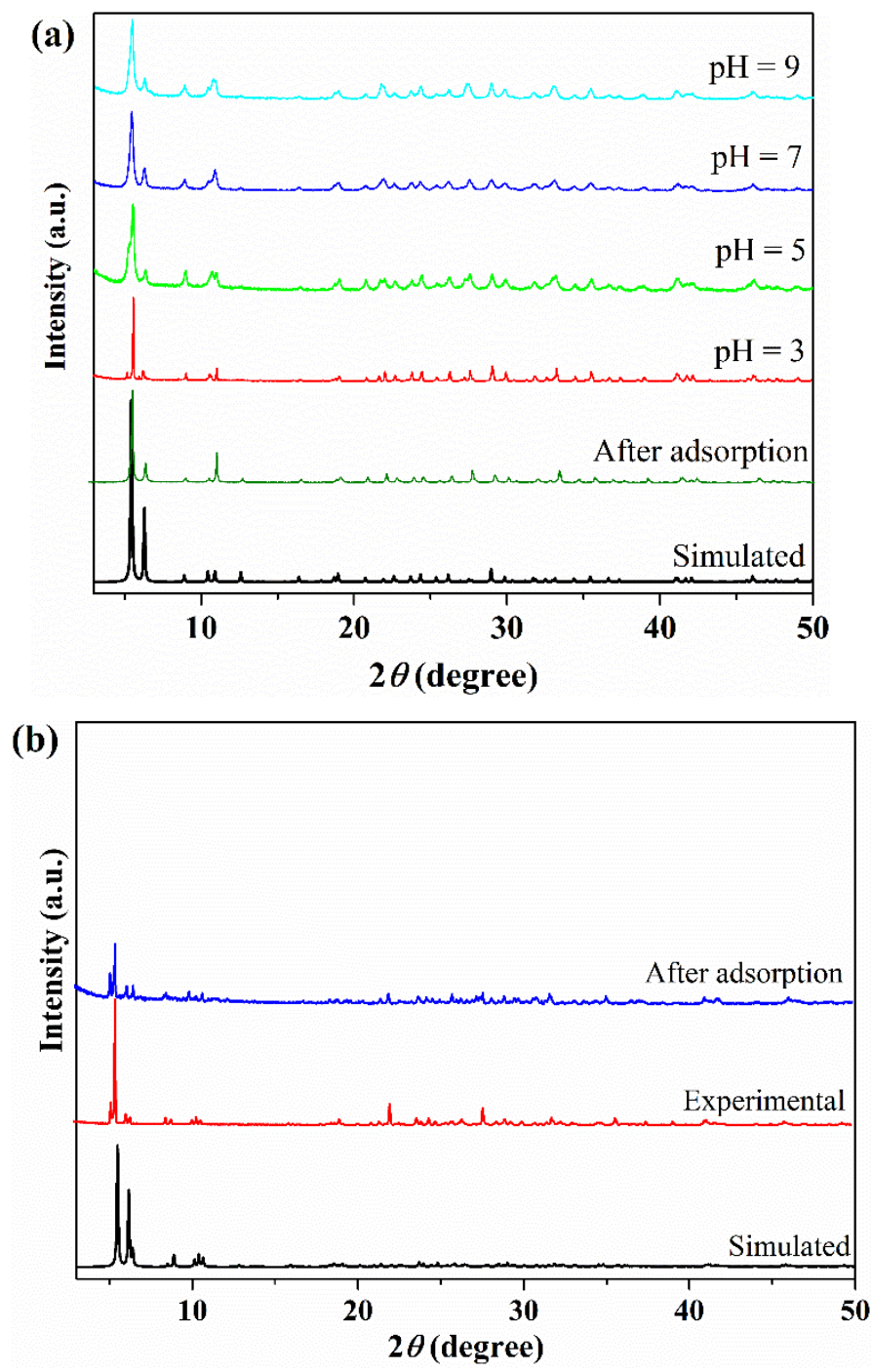

Figure S7. PXRD patterns for Th-TFBPDC (a) and Th-TFBPDC-i (b).

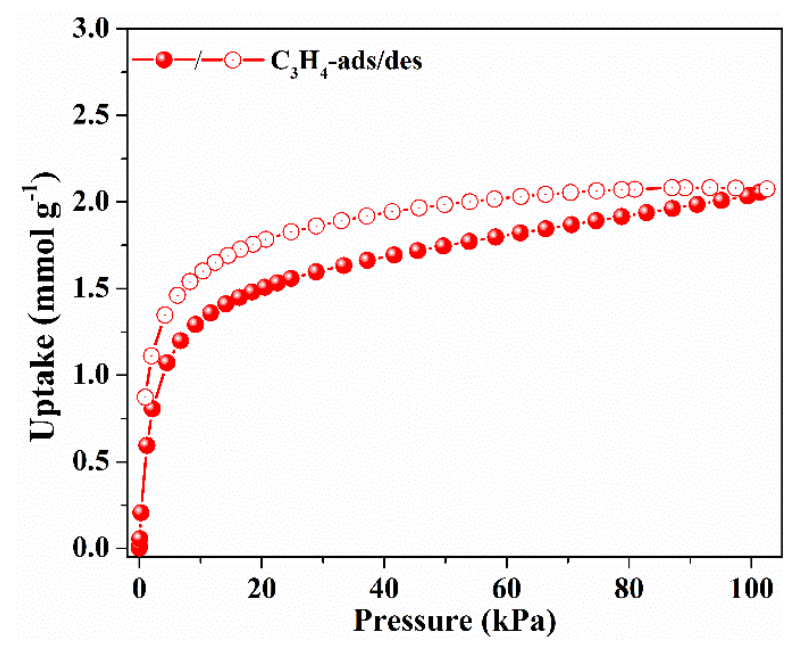

Figure S8. The $\mathrm{C}_{3} \mathrm{H}_{4}$ adsorption isotherms at $298 \mathrm{~K}$ for Th-TFBPDC-i. 

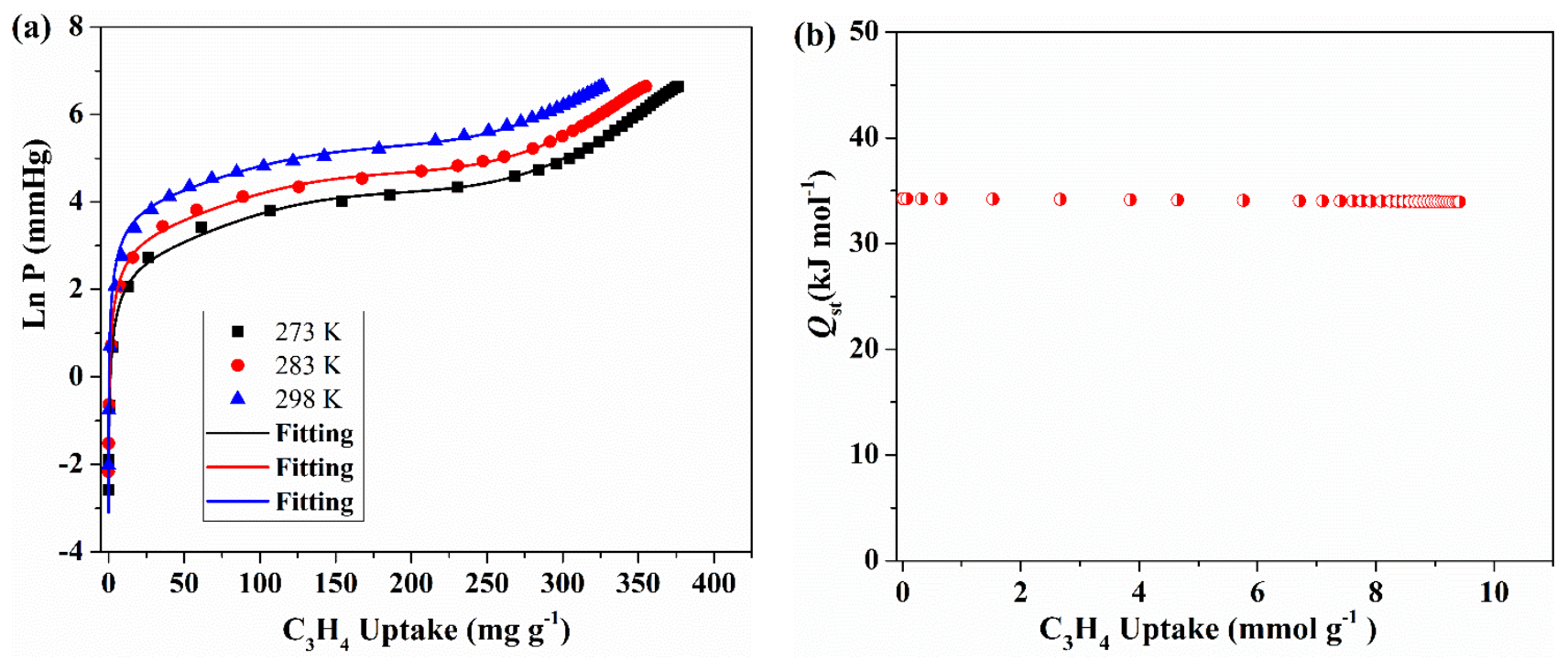

Figure S9. (a) Virial fits of $\mathrm{C}_{3} \mathrm{H}_{4}$ isotherms and (b) $Q_{\mathrm{st}}$ of $\mathrm{C}_{3} \mathrm{H}_{4}$ for Th-TFBPDC.

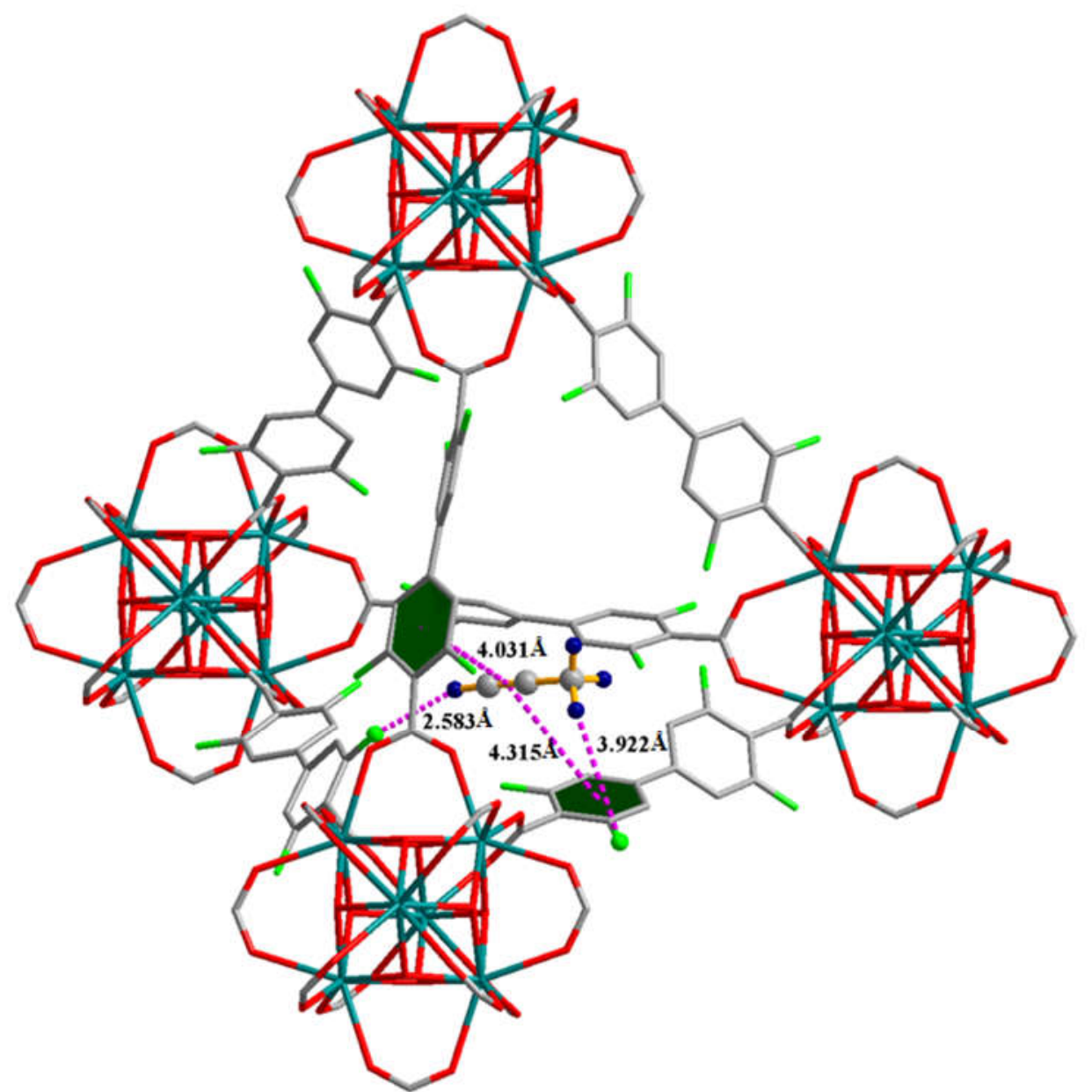

Figure S10. The $\mathrm{C}_{3} \mathrm{H}_{4}$ favorable adsorption binding sites in Th-TFBPDC. 
Table S1. Selected Bond Lengths ( $\AA$ ) of Th-TFBPDC and Th-TFBPDC-i.

\begin{tabular}{|c|c|c|c|c|c|c|c|}
\hline \multicolumn{2}{|c|}{ Th-TFBPDC } & \multicolumn{6}{|c|}{ Th-TFBPDC-i } \\
\hline Th1-O1A ${ }^{\mathrm{a}}$ & $2.389(5)$ & Th1-O2 & $2.343(7)$ & Th2-O3A & $2.357(6)$ & Th3-O2D & $2.357(5)$ \\
\hline Th1-O1B & $2.389(5)$ & Th1-O1 & $2.358(8)$ & Th2-O3D & $2.357(6)$ & Th3-O2 & $2.357(5)$ \\
\hline Th1-O1C & $2.389(5)$ & Th1-O3 & $2.382(6)$ & Th2-O1D & $2.385(6)$ & Th3-O3D & $2.364(6)$ \\
\hline Th1-O1 & $2.389(5)$ & Th1-O3A ${ }^{\mathrm{a}}$ & $2.382(6)$ & Th2-O1 & $2.385(6)$ & Th3-O3A & $2.364(6)$ \\
\hline Th1-O2D & $2.464(6)$ & Th1-O4 & $2.502(5)$ & Th2-O8 & $2.466(8)$ & Th3-O11 & $2.486(10)$ \\
\hline Th1-O2A & $2.464(6)$ & Th1-O4A & $2.502(5)$ & Th2-O5 & $2.486(5)$ & Th3-O7F & $2.476(5)$ \\
\hline Th1-O2E & $2.464(6)$ & Th1-O6B & $2.519(5)$ & Th2-O5E & $2.486(5)$ & Th3-O7G & $2.476(5)$ \\
\hline Th1-O2 & $2.464(6)$ & Th1-O6C & $2.519(5)$ & Th2-O9 & $2.533(9)$ & Th3-O10 & $2.432(8)$ \\
\hline Th1-O1W & $2.688(15)$ & Th1-O1W & $2.702(8)$ & $\mathrm{Th} 2-\mathrm{O} 2 \mathrm{~W}$ & $2.678(8)$ & Th3-O3W & $2.702(17)$ \\
\hline
\end{tabular}

Table S2. Comparison of the $\mathrm{C}_{3} \mathrm{H}_{4}$ uptakes at $298 \mathrm{~K}$ and $1 \mathrm{~atm}$ and adsorption enthalpies $\left(Q_{\text {st }}\right)$ (at zero-loading) for reported MOFs.

\begin{tabular}{lllll}
\hline MOF & BET $\left(\mathrm{m}^{2} \mathrm{~g}^{-1}\right)$ & $\begin{array}{l}\mathrm{C}_{3} \mathrm{H}_{4} \text { uptake } \\
\left(\mathrm{mmol} \mathrm{g}^{-1}\right)\end{array}$ & $\begin{array}{l}Q_{\text {st }}\left(\mathrm{C}_{3} \mathrm{H}_{4}\right) \\
\left(\mathrm{kJ} \mathrm{mol}^{-1}\right)\end{array}$ & Reference \\
\hline SIFSIX-1-Cu & 1128 & 8.76 & 37 & Ref. 2 \\
Th-TFBPDC & 1699 & $\mathbf{8 . 1 6}$ & 34.2 & This work \\
JXNU-6 & 856.3 & 5.07 & 39.9 & Ref. 3 \\
SIFSIX-2-Cu-i & 586 & 4.46 & 45 & Ref. 2 \\
Mg-Gallate & 475 & 3.75 & 66 & Ref. 4 \\
GeFSIX-dps-Cu & 382 & 3.7 & 71.6 & Ref. 5 \\
ZU-62 & 476 & 3.64 & 71 & Ref. 6 \\
UTSA-200 & 612 & 3.58 & 62.3 & Ref. 7 \\
NKMOF-1-Ni & 382 & 3.5 & 65.1 & Ref. 8 \\
NKMOF-1-Cu & 374 & 3.3 & 67.2 & Ref. 8
\end{tabular}




$\begin{array}{lcccc}\text { Co-Gallate } & 559 & 3.21 & 82 & \text { Ref. } 4 \\ \text { Ca-based MOF } & 224 & 3.18 & 55.4 & \text { Ref. } 9 \\ \text { SIFSIX-3-Ni } & 351 & 2.98 & 68 & \text { Ref. } 2 \\ \text { ELM-12 } & 740 & 2.77 & 60 & \text { Ref. } 10 \\ \text { Ni-Gallate } & 424 & 2.65 & 84 & \text { Ref. } 4 \\ \text { SIFSIX-3-Zn } & 230 & 2.26 & 74.5 & \text { Ref. } 2\end{array}$

\section{References}

(1) Rowsell, J. L. C.; Yaghi, O. M. Effects of Functionalization, Catenation, and Variation of the Metal Oxide and Organic Linking Units on the Low-Pressure Hydrogen Adsorption Properties of Metal-Organic Frameworks. J. Am. Chem. Soc. 2006, 128, 1304-1315.

(2) Yang, L.; Cui, X.; Yang, Q.; Qian, S.; Wu, H.; Bao, Z.; Zhang, Z.; Ren, Q.; Zhou, W.; Chen, B; Xing, H. A Single-Molecule Propyne Trap: Highly Efficient Removal of Propyne from Propylene with Anion-Pillared Ultramicroporous Materials. Adv. Mater. 2018, 1705374.

(3) Lin, Z.-T.; Liu, Q.-Y.; Yang, L.; He, C.-T.; Li, L.; Wang, Y.-L. Fluorinated Biphenyldicarboxylate -Based Metal-Organic Framework Exhibiting Efficient Propyne/Propylene Separation. Inorg. Chem. 2020, 59, 4030-4036.

(4) Li, Z.; Li, L.; Guo, L.; Wang, J.; Yang, Q.; Zhang, Z.; Yang, Y.; Bao, Z.; Ren, Q. Gallate-Based Metal-Organic Frameworks for Highly Efficient Removal of Trace Propyne from Propylene. Ind. Eng. Chem. Res. 2020, 59, 13716-13723.

(5) Ke, T.; Wang, Q.; Shen, J.; Zhou, J.; Bao, Z.; Yang, Q.; Ren, Q. Molecular Sieving of C2-C3 Alkene from Alkyne with Tuned Threshold Pressure in Robust Layered Metal-Organic Frameworks. Angew. Chem. Int. Ed. 2020, 59, 12725-12730.

(6) Yang, L.; Cui, X.; Zhang, Z.; Yang, Q.; Bao, Z.; Ren, Q.; Xing, H. An Asymmetric Anion-Pillared Metal-Organic Framework as a Multisite Adsorbent Enables Simultaneous Removal of Propyne and Propadiene from Propylene. Angew. Chem. Int. Ed. 2018, 57, $13145-13149$.

(7) Li, L.; Wen, H.-M.; He, C.; Lin, R.-B.; Krishna, R.; Wu, H.; Zhou, W.; Li, J.; Li, B.; Chen, B. A Metal-Organic Framework with Suitable Pore Size and Specific Functional Sites for the Removal of Trace Propyne from Propylene. Angew. Chem. Int. Ed. 2018, 57, 15183-15188. 
(8) Peng, Y.-L.; He, C.; Pham, T.; Wang, T.; Li, P.; Krishna, R.; Forrest, K. A.; Hogan, A.; Suepaul, S.; Space, B.; Fang, M.; Chen, Y.; Zaworotko, M. J.; Li, J.; Li, L.; Zhang, Z.; Cheng, P.; Chen, B. Robust Microporous Metal-Organic Frameworks for Highly Efficient and Simultaneous Removal of Propyne and Propadiene from Propylene. Angew. Chem. Int. Ed. 2019, 58, $10209-10214$.

(9) Li, L.; Guo, L.; Zheng, F.; Zhang, Z.; Yang, Q.; Yang, Y.; Ren, Q.; Bao, Z. Calcium-Based Metal-Organic Framework for Simultaneous Capture of Trace Propyne and Propadiene from Propylene. ACS. Appl. Mater. Interfaces. 2020, 12, 17147-17154.

(10) Li, L.; Lin, R.-B.; Krishna, R.; Wang, X.; Li, B.; Wu, H.; Li, J.; Zhou, W.; Chen, B. Flexible-Robust Metal-Organic Framework for Efficient Removal of Propyne from Propylene. $J$. Am. Chem. Soc. 2017, 139, 7733-7736. 